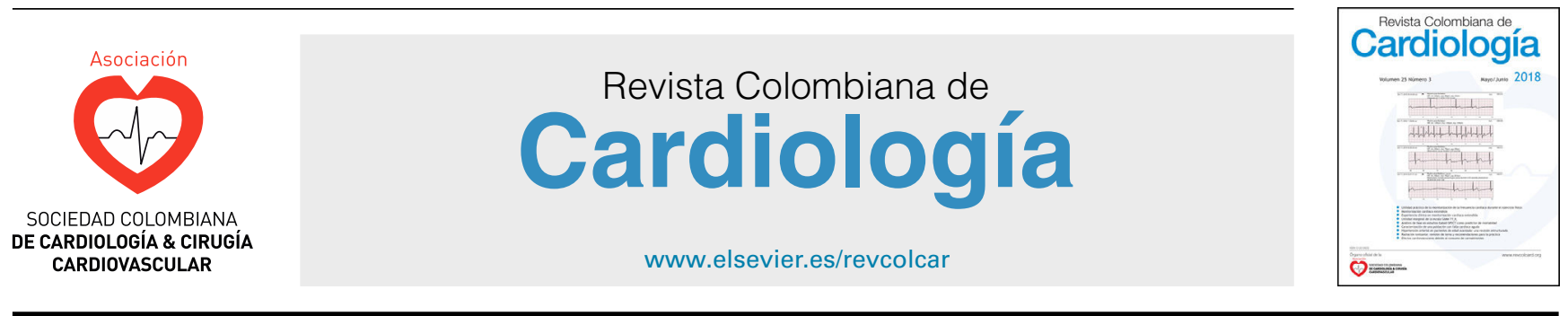

\title{
EDITORIAL
}

\section{Celebración del Día Mundial del Corazón en época de pandemia}

\section{Celebrating World Heart Day in a time of pandemic}

\author{
Darío Echeverri
}

Editor en Jefe. Revista Colombiana de Cardiología

La Federación Mundial del Corazón, con el apoyo de la Organización Mundial de la Salud (OMS) y La Organización de las Naciones Unidas para la Educación, la Ciencia y la Cultura (UNESCO), han designado desde el año 2000, el día 29 de septiembre como el "Día Mundial del Corazón". Esto obedece a la necesidad sentida de dar a conocer a la comunidad, en forma general y masiva, la importancia de las enfermedades cardiovasculares y su impacto en la salud mundial, e incentivar estrategias para su prevención, control y tratamiento. Desde entonces, en colaboración con la OMS, la Federación Mundial del Corazón organiza cada año en más de cien países, eventos que ayudan a adquirir conciencia sobre enfermedades cardiovasculares, como infarto agudo del miocardio, accidente cerebrovascular, hipertensión arterial y otras condiciones que afecten el miocardio y las arterias, ya que son, infortunadamente, las más mortíferas del mundo, pues no en vano generan 17,8 millones de muertes al año.

La Organización Panamericana de la Salud (OPS) también reconoce la importancia de impulsar políticas efectivas para reducir la ingesta de sal, fomentar la actividad física y continuar avanzando en la prevención y el control del tabaquismo. Estas son algunas de las medidas que promueve el Plan de Acción Regional para la prevención y el control de las enfermedades no transmisibles. Reducir la mortalidad por enfermedades cardiovasculares resulta imprescindible para lograr minimizar en un $25 \%$ la mortalidad prematura por enfermedades no transmisibles para el año 2025, según

Correo electrónico: revista@scc.org.co el compromiso adquirido en el Plan Mundial de Prevención y Control de Enfermedades no Transmisibles.

\section{Importancia de la prevención cardiovascular}

Una gran proporción de estas muertes podría evitarse si se hiciera consulta médica temprana para identificar y tratar factores de riesgo cardiovascular mediante una alimentación saludable, en la que haya reducción en la ingestión de sal, se incluya el ejercicio físico rutinario y se evite el consumo de tabaco. Promover y facilitar estos estilos de vida saludable requiere condiciones que faciliten el acceso a alimentos frescos y naturales, así como entornos seguros para la actividad física y la promoción de una vida libre de humo de tabaco.

Algunas de las acciones que se pueden emprender para cuidar el corazón, y que debemos trasmitir a nuestros pacientes y sus familias, se basan en recomendaciones tan sencillas como cocinar y comer sano (dieta mediterránea escaso consumo de grasas saturadas, aumento de la ingesta de ácidos omega 3 y pescado), procurar una dieta saludable con una cantidad menor al $10 \%$ de ingesta de energía proveniente de ácidos grasos saturados, consumir más de 200 gramos de frutas frescas al día, ingerir pescado 1 a 2 veces por semana, practicar ejercicio físico (150 minutos/semana de intensidad moderada o 75 minutos de actividad física aeróbica de intensidad vigorosa), limitar el consumo de sustancias perjudiciales como la sal (menos de $5 \mathrm{~g}$ al día), el tabaco o el alcohol (máximo $20 \mathrm{~g} /$ día en hombres o 2 copas y $10 \mathrm{~g} /$ día en mujeres o 1 copa), revisar periódicamente los niveles de colesterol sérico (todo el perfil de lípidos), 
y controlar el peso y el perímetro abdominal, entre muchas otras.

Desde hace ya varias décadas, ha venido documentándose cómo en países de altos ingresos, que han adoptado programas de prevención cardiovascular y, obviamente, mejores sistemas de salud para el diagnóstico y tratamiento oportuno de las enfermedades cardiovascular (y sus manifestaciones agudas, tales con infarto agudo del miocardio y el accidente cerebrovascular), han logrado reducir no solo la incidencia del infarto, sino también la mortalidad por enfermedades cardiovasculares hasta en un 31\%. Desde las publicaciones de Ford y colaboradores en el 2007, ha venido describiéndose cómo la reducción de la mortalidad se le puede atribuir hasta en un $44 \%$ a los cambios en los factores de riesgo y a la disminución del colesterol total, de la presión arterial sistólica, de la prevalencia del tabaquismo y de la inactividad física. Esto explica por qué recientemente la American Heart Association insistió en implementar el programa de "My life check- life's simple 7", que busca actuar sobre siete condiciones que pueden mantener un corazón sano: ser activo, mantener un peso saludable, aprender sobre las cifras de colesterol óptimas, no fumar, comer una dieta saludable para el corazón, mantener cifras de presión arterial saludables y conocer acerca de los niveles de glicemia y de la identificación y tratamiento temprano de la diabetes mellitus.

\section{Creación de entornos saludables}

El Día Mundial del Corazón insiste este año 2020 en la "creación de entornos saludables", como una estrategia necesaria y adicional para reducir los factores de riesgo asociados a las enfermedades cardiovasculares.

En la década del setenta, importantes episodios de contaminación del aire y el agua llevaron a la Agencia de Protección Ambiental (EPA) de los Estados Unidos a la adopción de leyes nacionales enfocadas en el control de la contaminación del aire, el agua y el suelo. A principios del siglo $x x$, las agencias de salud pública se enfocaron en eliminar las principales enfermedades infecciosas. Los esfuerzos para mejorar el saneamiento, el tratamiento del agua potable, las inspecciones de alimentos y el control de vectores dieron lugar a importantes avances en la salud y la calidad del medio ambiente. Estas acciones de mejoramiento en la salud y el bienestar humanos durante los últimos cincuenta años han sido extraordinarias. La esperanza de vida casi se ha duplicado y la mortalidad infantil se ha reducido notoriamente. Muchas de las enfermedades infecciosas antiguas han sido casi eliminadas. Estamos rodeados de innumerables dispositivos y comodidades que hacen nuestras vidas más fáciles que las de cualquier generación anterior.

No obstante, estas grandes ganancias han tenido un costo enorme, que apenas empezamos a comprender. Para lograr estas metas hemos estado despojando de manera insostenible a la tierra de sus recursos, degradando los ecosistemas del planeta y destruyendo nuestros sistemas de soporte vital. Una sociedad de consumo creciente y la destrucción y contaminación ambiental de las últimas décadas nos han llevado a un estado en el que ahora consumimos más de lo que nuestro planeta puede proporcionar de manera sostenible.
Para mejorar nuestra comodidad, estamos hipotecando la salud y el bienestar de nuestros hijos. Ahora, los amplios efectos ambientales son cada vez más evidentes y nuestra comprensión de la relación entre el medio ambiente y la salud humana está evolucionando. Mientras que la salud ambiental una vez se centró en la prevención de enfermedades infecciosas, ahora tener un medio ambiente saludable requerirá una visión más integral que la que se encuentra detrás de los enfoques regulatorios actuales.

El entorno construido en las estructuras hechas o modificadas por el hombre, que incluye los lugares donde trabajamos, comemos, descansamos, jugamos, calles y carreteras por las que viajamos, sistemas de servicios públi$\cos$ y sistemas de transporte, tiene un gran impacto en la salud cardiovascular. Generar no solo adecuados espacios físicos, sino procurar buenos ambientes laborales, hace parte de nuestra responsabilidad para el año 2020, en el Día Mundial del Corazón.

\section{Causas de muerte en el mundo}

Para el año 2004, según datos publicados por la World Health Organization en colaboración con la World Heart Federation y la World Stroke Organization, la enfermedad isquémica del corazón ocupaba el cuarto lugar, mientras que otras enfermedades cardiovasculares se ubicaban en el sexto lugar de mayor causa de enfermedad en el mundo. Se espera que para el año 2030 ambas condiciones suban al segundo y al cuarto lugar, respectivamente. Por otro lado, las enfermedades cardiovasculares constituyen la principal causa de mortalidad global y son un contribuyente importante a la reducción de la calidad de vida. En 2017, las enfermedades cardiovasculares causaron aproximadamente 17,8 millones de muertes en todo el mundo, lo que corresponde a 330 millones de años de vida perdidos y otros 35,6 millones de años vividos con discapacidad. La segunda causa fue el cáncer, que aportó otras 9.56 millones de personas (fig. 1). La suma de las dos supera las siguientes quince causas de mortalidad global. Vale la pena recordar que a medida que aumenta la edad de la población, incrementa la posibilidad de enfermedad cardiovascular y cáncer, y, en muchas ocasiones, coinciden ambas condiciones, lo cual agrava aún más el problema de salud.

Las tendencias en estos datos proporcionan indicadores muy importantes de las enfermedades cardiovasculares. Casi el $80 \%$ de las muertes por enfermedades cardiovasculares en todo el mundo ocurre en países de ingresos bajos y medianos, donde la carga de enfermedades cardiovasculares y factores de riesgo está aumentando. Aproximadamente, 48.000 personas mueren por enfermedades cardiovasculares cada día en todo el mundo, según las últimas investigaciones exhaustivas publicadas en 2017. En Colombia, las enfermedades cardiovasculares son la principal causa de muerte. En el 2019, murieron 61.300 personas por enfermedades cardiovasculares, cifra que corresponde aproximadamente a 170 personas diarias.

Finalmente, en época de pandemia por el virus SARS-CoV2-COVID-19, cuando todos los esfuerzos son dados por el personal de salud altamente comprometido y el gobierno nacional genera las mejores políticas en medio de la contingencia y el impacto económico y social, vemos como 


\section{Number of deaths by cause, World, 2017}

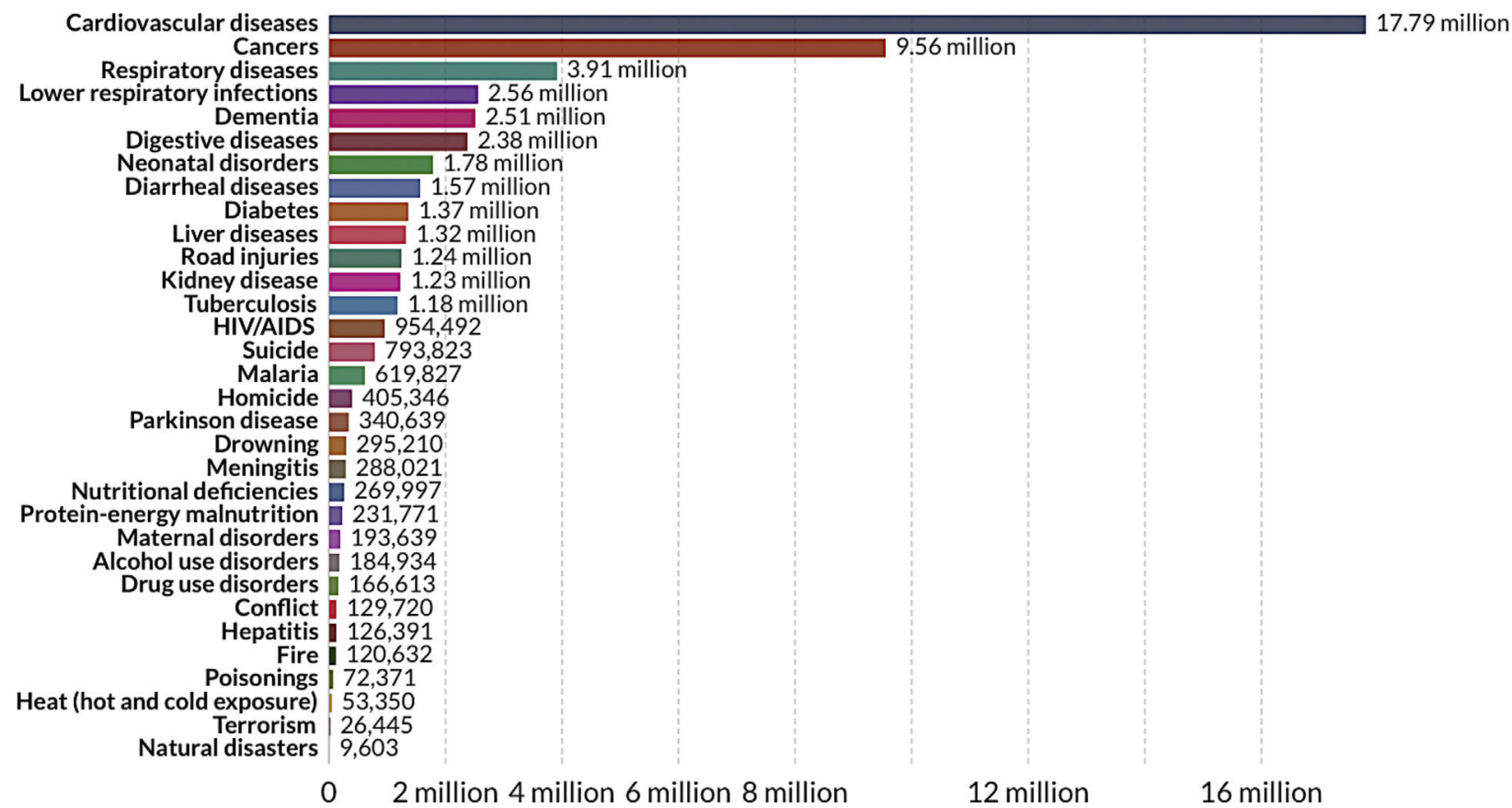

Source: IHME, Global Burden of Disease

Figura 1 Número de muertes por causa global en el año 2017. https: / / ourworldindata.org/causes-of-death.

el número de muertes reportadas en el mundo y en el país viene en aumento, pero no logra igualar o superar lo descrito por las enfermedades crónicas, en especial las cardiovasculares y el cáncer. Debemos hacerle frente común al COVID 19, pero no podemos descuidar la prevención, el diagnóstico oportuno y el tratamiento adecuado de las enfermedades cardiovasculares, como principal causa de morbi-mortalidad.

\section{Bibliografía recomendada}

1. Ford ES, Ajani UA, Croft JB, Critchley JA, Labarthe DR, Kottke TE, et al. Explaining the decrease in U.S. death from coronary disease, 1980-2000. N Engl J Med. 2007;356:2388-98.

2. Virani SS, Alonso A, Benjamin EJ, Bittencourt MS, Callaway CW, Carson AP, et al. Heart Disease and Stroke Statistics-2020 Update: A Report From the American Heart Association. Circulation. 2020;141:e139-596.

3. Costello A, Abbas M, Allen A, Ball S, Bell S, Bellamy R, et al. Managing the health effects of climate change: Lancet and University College London Institute for Global Health Commission. Lancet. 2009;373:1693-733.
4. Koehler K, Latshaw M, Matte T, Kass D, Frumkin H, Fox M, et al. Building healthy community environments: a public health approach. Public Health Rep. 2018;133 1 suppl:35S-43S.

5. Martin K, Landrigan PJ. Global health's grand challenge: a healthy planet and healthy people. Ann Glob Health. 2016;82:317-8.

6. Wierzbicka A, Pedersen E, Persson R, Nordquist B, Stålne K, Gao C. Healthy indoor environments: the need for a holistic approach. Int J Environ Res Public Health. 2018;15:1874.

7. GBD 2017 Causes of Death Collaborators. Global, regional, and national age-sex-specific mortality for 282 causes of death in 195 countries and territories, 1980-2017: a systematic analysis for the Global Burden of Disease Study 2017. Lancet. 2018;392:1736-88.

8. 2017 DALYs GBD, Collaborators HALE. Global, regional, and national disability-adjusted life-years (DALYs) for 359 diseases and injuries and healthy life expectancy (HALE) for 195 countries and territories, 1990-2017: a systematic analysis for the Global Burden of Disease Study 2017. Lancet. 2018;392:1859-922.

9. Mensah GA, Roth GA, Fuster V. The global burden of cardiovascular diseases and risk factors: 2020 and beyond. J Am Coll Cardiol. 2019;74:2529-32. 